\title{
Moving forward: early diagnosis of fetal spinal defects
}

Moving prenatal diagnosis and screening to earlier stages of pregnancy has probably been the most important progress in the field of Fetal Medicine in the recent years. Screening for chromosomal abnormalities is the most obvious example; the sensitivity of screening for Down syndrome by maternal age only (1980 s - early 1990 s) was only $30 \%$, which rose to $60 \%$ by second-trimester biochemical tests days as high as $95 \%$ with the implementation of first-trimester screening with ultrasound and biochemical markers [1].

A by-product of the advent of first-trimester screening for chromosomal abnormalities is that examiners became increasingly familiar with early fetal anatomy. As a result, nearly half of all major fetal structural defects can currently be detected at the 11-14 weeks' scan if a structured examination of the fetal anatomy is performed, and this includes virtually all cases of acrania, alobar holoprosencephaly, exomphalos, gastroschisis, megacystis and body stalk anomaly [2]. However, the (late 1980 s - early 1990s) and is nowa-

detection rate of spinal defects in early pregnancy has been notoriously lower than that, as only $14 \%$ of the cases with open spina bifida were picked up at the first-trimester scan in the Syngelaki et al. series [2].

These rates are very likely to improve through the examination of a novel firsttrimester ultrasound marker, the socalled intracranial translucency (IT). Intracranial translucency, which essentially represents the early fourth cerebral ventricle, was first described by Chaoui et al. in 2007 as an anechoic space between the brain stem and choroid plexus [3] ( $\odot$ Figure $1 \mathrm{~A})$.

In cases with spina bifida the fourth ventricle is compressed through caudal displacement of the brain stem, and IT is usually obliterated. Additional measurements and ratios of the posterior fossa have also been proposed in order to improve sensitivity [4 5], and abnormal measurements (obliteration or dilation) have been reported in various CNS de-

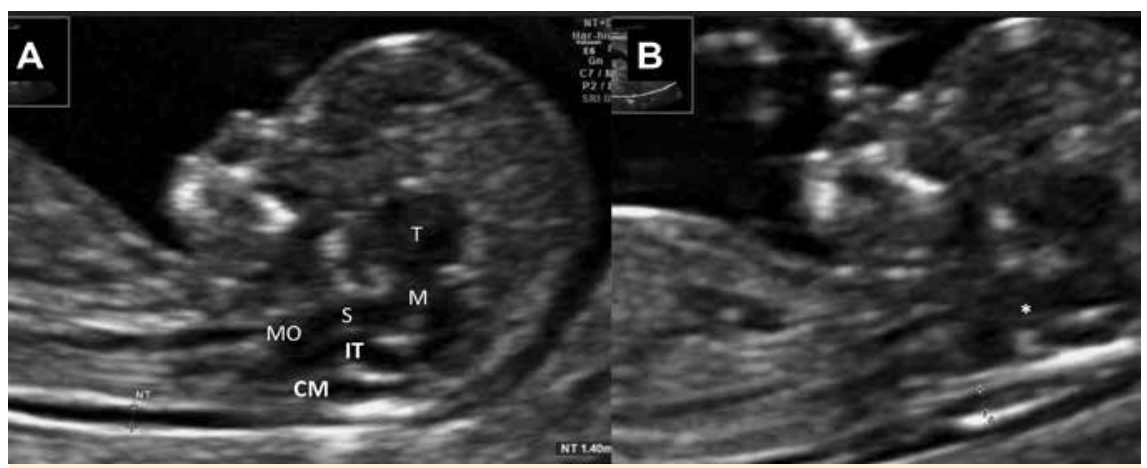

Fig. 1 Normal imaging of the first-trimester posterior fossa (A), and, obliteration of the normal intracranial translucency in an $11+3$ weeks' fetus with open spina bifida (B, marked with asterisk); T: thalamus; M: mesencephalon; S: brain stem; MO: medulla oblongata; IT: intracranial translucency; CM: cisterna magna fects, such as diastematomyelia [6], Dandy-Walker complex [7] or Blake's pouch cyst [8]. Similar to nuchal translucency, IT can be measured using a semi-automated algorithm, with high reproducibility [9]. A large prospective multicentre study in Germany very recently reported $100 \%$ sensitivity (11/11) for open spina bifida by structured examination of the first-trimester posterior fossa [10].

In the case of 0 Figure 1B, obliteration of the fetal IT at $11+2$ weeks (CRL $48 \mathrm{~mm}$ ) led to the identification of a lumbar defect measuring $5.2 \times 2.4 \mathrm{~mm}$ ( $\odot$ Figure $2 \mathrm{2A}, \mathbf{B}$ ) before the disruption in the architecture of the spine becoming visible (o Figure $2 \mathrm{C}$ ), in a couple with no known risk factors for CNS defect. The parents were counselled about the diagnosis and opted for termination of pregnancy at 12 weeks.

The rationale for early detection is that it offers the parents time to digest the news and realize their feelings, facilitates them in their decision-making process and lessens the degree of psychological and physical strain should they opt for a termination. One might argue that the earlier the diagnosis, the easier the parents might decide to terminate pregnancy, which for some might pose an ethical question. Although there is not nearly enough data for the case of structural defects, the paradigm of chromosomal abnormalities shows that gestational age at diagnosis mostly influences parents' decision for mild and moderate rather than severe ab- 


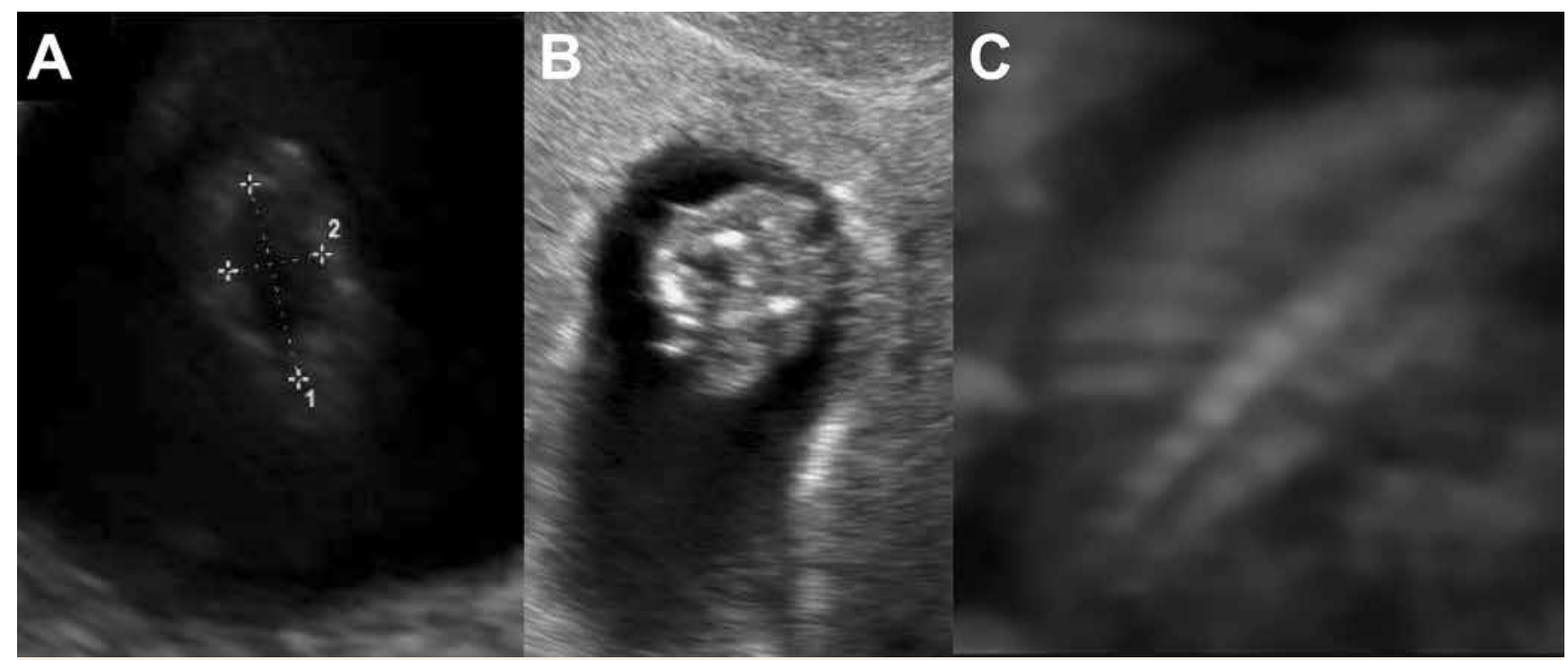

Fig. 2 Disruption of the continuity of the skin in the lumbar area in the fetus of Figure 1B, in a coronal plane (A) and axial plane (B), with apparently normal architecture of the lumbar spine on 3D reconstruction (C).

normalities [11]. Anyhow, moving earlier in pregnancy appears to be the way forward, and, as many experts believe, a combination of maternal characteristics and history together with specific biophysical and biochemical tests in the first trimester will enable providers to predict many pregnancy complications [12] and produce a triage model which will result in tailored subsequent obstetric care.

\section{References}

1 Benn P, Cuckle H, Pergament E. Non-invasive prenatal testing for aneuploidy: current status and future prospects. Ultrasound in obstetrics \& gynecology : the official journal of the International Society of Ultrasound in Obstetrics and Gynecology 2013; 42: 15-33

2 Syngelaki A, Chelemen T, Dagklis T et al. Challenges in the diagnosis of fetal non-chromosomal abnormalities at 11-13 weeks. Prenatal diagnosis 2011; 31: 90-102

3 Chaoui R, Benoit B, Mitkowska-Wozniak H et al. Assessment of intracranial translucency (IT) in the detection of spina bifida at the 1113-week scan. Ultrasound in obstetrics \& gynecology : the official journal of the International Society of Ultrasound in Obstetrics and Gynecology 2009; 34: 249-252
4 Chaoui $R$, Benoit B, Heling KS et al. Prospective detection of open spina bifida at 11-13 weeks by assessing intracranial translucency and posterior brain. Ultrasound in obstetrics \& gynecology : the official journal of the International Society of Ultrasound in Obstetrics and Gynecology 2011; 38: 722-726

5 Karl K, Heling KS, Chaoui R. Fluid area measurements in the posterior fossa at 11-13 weeks in normal fetuses and fetuses with open spina bifida. Fetal diagnosis and therapy $2015 ; 37: 289-293$

6 Eleftheriades M, Grigoriadis C, Sotiriadis A et al. Intracranial translucency and spinal cord defects: early prenatal diagnosis of diastematomyelia. Journal of ultrasound in medicine : official journal of the American Institute of Ultrasound in Medicine 2013; 32: 1676-1677

7 Volpe P, Contro E, Fanelli T et al. Appearance of the fetal posterior fossa at 11-14 weeks in foetuses with Dandy-Walker complex or chromosomal anomalies. Ultrasound in obstetrics \& gynecology : the official journal of the International Society of Ultrasound in Obstetrics and Gynecology 2015; doi: 10. 1002/uog. 14883

8 Tonni G, Grisolia G. Dilated intracranial translucency and Blake's pouch cyst: firsttrimester ultrasound markers of occipital cephalocele diagnosed using novel three-dimensional reslicing technique. Journal of clinical ultrasound : JCU 2014; 42: 157-161
9 Karl K, Kagan KO, Chaoui R. Intra- and interoperator reliability of manual and semi-automated measurements of intracranial translucency. Ultrasound in obstetrics \& gynecology : the official journal of the International Society of Ultrasound in Obstetrics and Gynecology 2012; 39: 164-168

10 Chen FC, Gerhardt J, Entezami M et al. Detection of Spina Bifida by First Trimester Screening - Results of the Prospective Multicenter Berlin IT-Study. Ultraschall in der Medizin 2015; doi: 10. 1055/s-0034-1399483

11 Evans MI, Sobiecki MA, Krivchenia EL et al. Parental decisions to terminate/continue following abnormal cytogenetic prenatal diagnosis: „what" is still more important than „when“. American journal of medical genetics 1996; 61: 353-355

12 Nicolaides $K H$. Turning the pyramid of prenatal care. Fetal diagnosis and therapy 2011; 29: 183-196

\section{Dr. Alexandros Sotiriadis}

Assistant Professor in Obstetrics and Gynecology

Aristotle University of Thessaloniki, Greece Email: asotir@gmail.com 